\title{
Generalized Stirling transform
}

\author{
Mourad Rahmani
}




\title{
GENERALIZED STIRLING TRANSFORM
}

\author{
MOURAD RAHMANI
}

Received 17 December, 2013

\begin{abstract}
In this paper algorithms are developed for computing the Stirling transform and the inverse Stirling transform; specifically, we investigate a class of sequences satisfying a twoterm recurrence. We derive a general identity which generalizes the usual Stirling transform and investigate the corresponding generating functions also. In addition, some interesting consequences of these results related to classical sequences like Fibonacci, Bernoulli and the numbers of derangements have been derived.
\end{abstract}

2010 Mathematics Subject Classification: 05A19; 11B68

Keywords: Bernoulli polynomials, Fibonacci numbers, Hankel transform, two-term recurrence, Stirling transform

\section{INTRODUCTION}

The Stirling numbers arise frequently in mathematics, especially in enumerative problems. This is the reason of their important role in combinatorial analysis, number theory, probability, graph theory, calculus of finite differences and interpolation. The notations for these numbers have never been standardized, this paper follows the notation of Riordan for the signed Stirling numbers of the first kind $s(n, k)$ and Knuth's notation for the Stirling numbers of the second kind $\left\{\begin{array}{l}n \\ k\end{array}\right\}$.

The Stirling transform of a sequence $\left(a_{n}\right)$ is the the sequence $\left(b_{n}\right)$ given by

$$
b_{n}=\sum_{k=0}^{n}\left\{\begin{array}{l}
n \\
k
\end{array}\right\} a_{k},
$$

and the inverse transform is

$$
a_{n}=\sum_{k=0}^{n} s(n, k) b_{k} .
$$

The identity (1.1) has a combinatorial interpretation given in [2]. If $a_{n}$ is the number of objects in some class with points labeled $1,2, \ldots, n$ (with all labels distinct) then $b_{n}$ is the number of objects with points labeled $1,2, \ldots, n$ (with repetitions allowed). 
In this paper algorithms are developed for computing the Stirling transform and the inverse Stirling transform; specifically, we investigate a class of sequences satisfying a two-term recurrence. We derive a general identity which generalizes the usual Stirling transform and investigate the corresponding generating functions also.

Given a sequence $a_{m}:=a_{0, m}(m \geq 0)$. We construct an infinite matrix $\delta:=$ $\left(a_{n, m}\right)$ as follows:

The first row $a_{0, m}$ of the matrix is the initial sequence; the first column $b_{n}:=a_{n, 0}$ $(n \geq 0)$ is called the final sequence and, each entry $a_{n, m}$ is given recursively by

$$
a_{n+1, m}=a_{n, m+1}+m a_{n, m} .
$$

Conversely, if we start with the final sequence, the matrix $\delta$ can be recovered by the recursive relations

$$
a_{n, m+1}=a_{n+1, m}-m a_{n, m}
$$

\section{DEFINITIONS AND NOTATION}

In this section, we introduce some definitions and notations which are useful in the rest of the paper. $\mathbb{N}$ being the set of positive integers and $\mathbb{N}_{0}=\mathbb{N} \cup\{0\}$.

The falling and rising factorials are defined, respectively by

$$
(x)_{n}=x(x-1) \cdots(x-n+1),(x)_{0}=1
$$

and

$$
\langle x\rangle_{n}=x(x+1) \cdots(x+n-1),\langle x\rangle_{0}=1 .
$$

The (signed) Stirling numbers $s(n, k)$ of the first kind, are usually defined by

$$
(x)_{n}=\sum_{k=0}^{n} s(n, k) x^{k},
$$

or by the following generating function

$$
\frac{1}{k !}(\ln (1+x))^{k}=\sum_{n \geq k} s(n, k) \frac{x^{n}}{n !} .
$$

It follows from (2.1) or (2.2) that

$$
s(n+1, k)=s(n, k-1)-n s(n, k)
$$

and that

$$
s(n, 0)=\delta_{n, 0} \quad(n \in \mathbb{N}), s(n, k)=0 \quad(k>n \text { or } k<0),
$$

where $\delta_{n, m}$ denotes the Kronecker symbol. 
The Stirling numbers $\left\{\begin{array}{l}n \\ k\end{array}\right\}$ of the second kind count the number of possible partitions of a set of $n$ objects into $k$ disjoint blocks. These numbers can be defined explicitly by

$$
x^{n}=\sum_{k=0}^{n}\left\{\begin{array}{l}
n \\
k
\end{array}\right\}(x)_{k} .
$$

For any positive $r \in \mathbb{N}$ the quantity $\left\{\begin{array}{l}n \\ k\end{array}\right\}_{r}$ denotes the number of partitions of a set of $n$ objects into exactly $k$ nonempty, disjoint subsets, such that the first $r$ elements are in distinct subsets. These numbers obey the recurrence relation

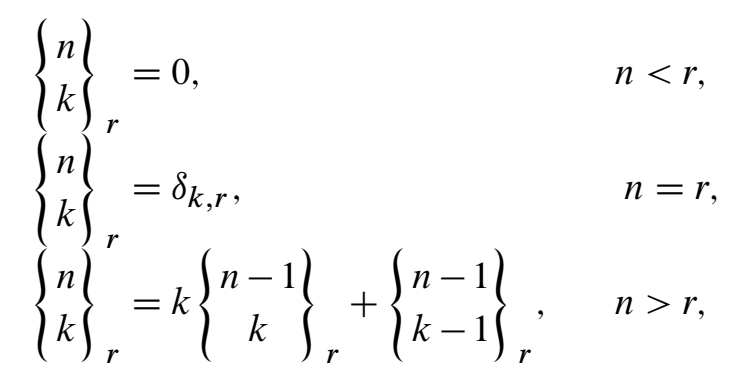

The exponential generating function is given by

$$
\sum_{n \geq k}\left\{\begin{array}{l}
n+r \\
k+r
\end{array}\right\} \frac{x^{n}}{n !}=\frac{1}{k !} e^{r x}\left(e^{x}-1\right)^{k} .
$$

The properties

$$
\left\{\begin{array}{l}
n \\
r
\end{array}\right\}_{r}=r^{n-r}
$$

and

$$
\left\{\begin{array}{l}
n+r \\
k+r
\end{array}\right\}_{r}=\left\{\begin{array}{l}
n+r \\
k+r
\end{array}\right\}_{r-1}-(r-1)\left\{\begin{array}{c}
n+r-1 \\
k+r
\end{array}\right\}_{r-1}
$$

are given in [3], which one can consult for more details on $r$-Stirling numbers.

\section{COMBINATORIAL IDENTITIES}

Theorem 1. Given an initial sequence $\left(a_{0, m}\right)_{m \geq 0}$, define the matrix $\&$ by (1.3). Then, the entries of the infinite matrix 8 are given by

$$
a_{n, m}=\sum_{k=0}^{n}\left\{\begin{array}{l}
n+m \\
k+m
\end{array}\right\}_{m} a_{0, m+k} .
$$


Proof. We prove by induction on $n$, the result clearly holds for $n=0$. By induction hypothesis

$$
\begin{aligned}
a_{n, m+1}+m a_{n, m} & =\sum_{k=0}^{n}\left\{\begin{array}{l}
n+m+1 \\
k+m+1
\end{array}\right\}_{m+1} a_{0, m+k+1}+m\left\{\begin{array}{c}
n+m \\
m
\end{array}\right\}_{m} a_{0, m} \\
& +m \sum_{k=1}^{n-1}\left\{\begin{array}{l}
n+m \\
k+m
\end{array}\right\}_{m} a_{0, m+k} \\
& =\sum_{k=0}^{n}\left\{\begin{array}{l}
n+m+1 \\
k+m+1
\end{array}\right\}_{m+1} a_{0, m+k+1}+m\left\{\begin{array}{c}
n+m \\
m
\end{array}\right\}_{m} a_{0, m} \\
& +m \sum_{k=0}^{n-2}\left\{\begin{array}{c}
n+m \\
k+m+1
\end{array}\right\}_{m} a_{0, m+k+1} \\
& =\left\{\begin{array}{l}
n+m+1 \\
n+m+1
\end{array}\right\}_{m+1} a_{0, m+n+1}+\left\{\begin{array}{c}
n+m+1 \\
n+m
\end{array}\right\}_{m+1} a_{0, m+n} \\
& +\sum_{k=0}^{n-2}\left\{\begin{array}{l}
n+m+1 \\
k+m+1
\end{array}\right\}_{m+1} a_{0, m+k+1} \\
& +m\left\{\begin{array}{c}
n+m \\
m
\end{array}\right\}_{m} a_{0, m}+m \sum_{k=0}^{n-2}\left\{\begin{array}{c}
n+m \\
k+m+1
\end{array}\right\}_{m} a_{0, m+k+1} .
\end{aligned}
$$

From (2.6) and after some rearrangements, we get

$$
\begin{aligned}
a_{n, m+1}+m a_{n, m} & =\sum_{k=0}^{n+1}\left\{\begin{array}{c}
n+m+1 \\
k+m
\end{array}\right\}_{m} a_{0, m+k} . \\
& =a_{n+1, m} .
\end{aligned}
$$

Theorem 2. Given a final sequence $\left(a_{n, 0}\right)_{n \geq 0}$, define the matrix 8 by (1.4). Then, the entries of the infinite matrix $\&$ are given by

$$
a_{n, m}=\sum_{k=0}^{m} s(m, k) a_{n+k, 0}
$$

Proof. We prove by induction on $m$, the result clearly holds for $n=0$. By induction hypothesis and (2.3), we have

$$
a_{n+1, m}-m a_{n, m}=\sum_{k=1}^{m+1} s(m, k-1) a_{n+k, 0}-m \sum_{k=0}^{m} s(m, k) a_{n+k, 0}
$$




$$
\begin{aligned}
& =s(m, m) a_{n+m+1,0}+\sum_{k=1}^{m} s(m, k-1) a_{n+k, 0} \\
& -m s(m, 0) a_{n, 0}-m \sum_{k=1}^{m} s(m, k) a_{n+k, 0} \\
& =s(m, m) a_{n+m+1,0}+\sum_{k=1}^{m}(s(m, k-1)-m s(m, k)) a_{n+k, 0} \\
& -m s(m, 0) a_{n, 0} \\
& =a_{n, m+1}
\end{aligned}
$$

Corollary 1. For $n, m \in \mathbb{N}_{0}$, we have

$$
\sum_{k=0}^{m} s(m, k) b_{n+k}=\sum_{k=0}^{n}\left\{\begin{array}{l}
n+m \\
k+m
\end{array}\right\}_{m} a_{m+k} .
$$

The last identity can be viewed as the generalized Stirling transform which reduced, for $m=0$, to the Stirling transform (1.1) of the sequence $a_{n}$, and for $n=0$ reduces to the inverse Stirling transform (1.2) of the sequence $b_{m}$. We may now formulate the following algorithms

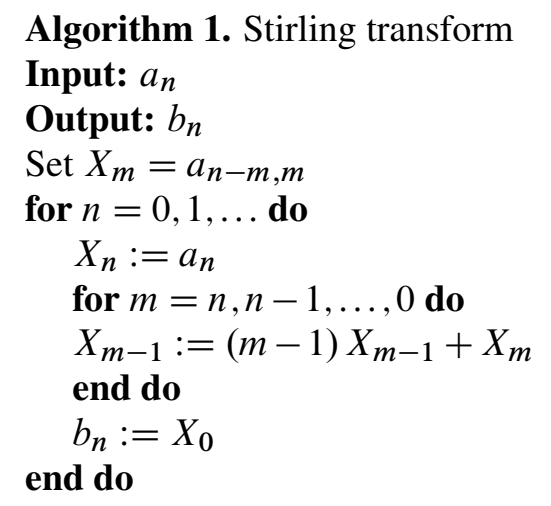

Algorithm 2. inverse Stirling transform

Input: $b_{m}$

Output: $a_{m}$

Set $Y_{n}=b_{n, m-n}$

for $m=0,1, \ldots$ do

$Y_{m}:=b_{m}$

for $n=m, m-1, \ldots, 0$ do 


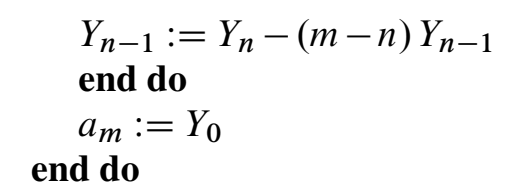

Example 1. Setting $a_{0, m}=1$ in (3.3), we get the well known identity [7]

$$
\sum_{k=0}^{n}\left\{\begin{array}{l}
n+m \\
k+m
\end{array}\right\}_{m}=\sum_{k=0}^{m} s(m, k) B_{n+k}
$$

where $B_{n}$ is the $n$th Bell number.

Example 2. Let $\left(F_{n}\right)_{n \in \mathbb{N}_{0}}$ be the Fibonacci sequence given by Binet's formula

$$
F_{n}=\frac{1}{\sqrt{5}}\left(\alpha^{n}-\beta^{n}\right)
$$

where $\alpha=\frac{1+\sqrt{5}}{2}$ and $\beta=\frac{1-\sqrt{5}}{2}$. If the initial sequence

$$
a_{0, m}=\frac{(-1)^{m}}{\sqrt{5}}\left(\langle-\alpha\rangle_{m}-\langle-\beta\rangle_{m}\right),
$$

then we get the following matrix

$$
8=\left(\begin{array}{cccccccc}
0 & 1 & 0 & 1 & -4 & 19 & -108 & \cdots \\
1 & 1 & 1 & -1 & 3 & -13 & 71 & \\
1 & 2 & 1 & 0 & -1 & 6 & -37 & \\
2 & 3 & 2 & -1 & 2 & -7 & 34 & \\
3 & 5 & 3 & -1 & 1 & -1 & -3 & \\
5 & 8 & 5 & -2 & 3 & -8 & 31 & \\
8 & 13 & 8 & -3 & 4 & -9 & 28 & \\
13 & 21 & 13 & -5 & 7 & -17 & 59 & \\
\vdots & & & & & &
\end{array}\right)
$$

From this matrix we observe that $a_{n, 0}=a_{n, 2}=-a_{n+2,3}=F_{n}$, and $a_{n+3,4}=L_{n}$, where $\left(L_{n}\right)_{n \in \mathbb{N}_{0}}$ the Lucas sequence given by Binet's formula

$$
L_{n}=\alpha^{n}+\beta^{n} .
$$

It is well known that the $F_{n}$ and $L_{n}$ are connected by the formula

$$
L_{n}=F_{n-1}+F_{n+1}, \quad(n \in \mathbb{N}) \text {. }
$$

By (3.3), one can deduce that

$$
\sum_{k=0}^{m} s(m, k) F_{n+k}=\frac{1}{\sqrt{5}} \sum_{k=0}^{n}(-1)^{m+k}\left\{\begin{array}{l}
n+m \\
k+m
\end{array}\right\}_{m}\left(\langle-\alpha\rangle_{m+k}-\langle-\beta\rangle_{m+k}\right)
$$


and by Theorem 2, we get

$$
\begin{aligned}
F_{n} & =\sum_{k=0}^{2} s(2, k) F_{n+k}=-F_{n+1}+F_{n+2} \\
& =-\sum_{k=0}^{3} s(3, k) F_{n+2+k}=-2 F_{n+3}+3 F_{n+4}-F_{n+5},
\end{aligned}
$$

and for $n \in \mathbb{N}_{0}$, we have

\section{Corollary 2.}

$$
L_{n}=\sum_{k=0}^{4} s(4, k) F_{n+3+k}=-6 F_{n+4}+11 F_{n+5}-6 F_{n+6}+F_{n+7} .
$$

By Theorem 1, one easily gets the following explicit formulas.

Corollary 3. For $n \in \mathbb{N}_{0}$, we have

$$
\begin{aligned}
F_{n} & =\frac{1}{\sqrt{5}} \sum_{k=0}^{n}(-1)^{k}\left\{\begin{array}{l}
n \\
k
\end{array}\right\}\left(\langle-\alpha\rangle_{k}-\langle-\beta\rangle_{k}\right) \\
& =\frac{1}{\sqrt{5}} \sum_{k=0}^{n}(-1)^{k}\left\{\begin{array}{l}
n+2 \\
k+2
\end{array}\right\}_{2}\left(\langle-\alpha\rangle_{k+2}-\langle-\beta\rangle_{k+2}\right) \\
& =\frac{1}{\sqrt{5}} \sum_{k=0}^{n+2}(-1)^{k}\left\{\begin{array}{l}
n+5 \\
k+3
\end{array}\right\}_{3}\left(\langle-\alpha\rangle_{k+3}-\langle-\beta\rangle_{k+3}\right)
\end{aligned}
$$

and

$$
L_{n}=\frac{1}{\sqrt{5}} \sum_{k=0}^{n+3}(-1)^{k}\left\{\begin{array}{l}
n+7 \\
k+4
\end{array}\right\}_{4}\left(\langle-\alpha\rangle_{k+4}-\langle-\beta\rangle_{k+4}\right) \text {. }
$$

\section{GENERATING FUNCTION}

Theorem 3. Suppose that the initial sequence $a_{0, m+r}$ has the following exponential generating function $A_{r}(z)=\sum_{k \geq 0} a_{0, k+r} \frac{z^{k}}{k !}$. Then the sequence $\left\{a_{n, r}\right\}_{n}$ of the $r$ th columns of the matrix 8 has an exponential generating function $\mathcal{B}_{r}(z)=\sum_{n \geq 0} a_{n, r} \frac{z^{n}}{n !}$ given by

$$
B_{r}(z)=e^{r z} A_{r}\left(e^{z}-1\right)
$$


Proof. We have

$$
\begin{aligned}
B_{r}(z) & =\sum_{k \geq 0} a_{0, r+k} \sum_{n \geq 0}\left\{\begin{array}{l}
n+r \\
k+r
\end{array}\right\}_{r} \frac{z^{n}}{n !} \\
& =\sum_{k \geq 0} a_{0, r+k} \frac{1}{k !} e^{r z}\left(e^{z}-1\right)^{k} \\
& =e^{r z} \sum_{k \geq 0} a_{0, r+k} \frac{\left(e^{z}-1\right)^{k}}{k !} \\
& =e^{r z} A_{r}\left(e^{z}-1\right) .
\end{aligned}
$$

Theorem 4. Suppose that the final sequence $a_{n+r, 0}$ has the following exponential generating function $\mathcal{B}_{r}(z)=\sum_{k \geq 0} a_{k+r, 0} \frac{z^{k}}{k !}$. Then the sequence $\left\{a_{r, m}\right\}_{m}$ of the $r$ th rows of the matrix $\&$ has an exponential generating function $\mathcal{A}_{r}(z)=\sum_{m \geq 0} a_{r, m} \frac{z^{m}}{m !}$ given by

$$
\mathcal{A}_{r}(z)=\mathscr{B}_{r}(\ln (1+z))
$$

Proof. We have

$$
\begin{aligned}
\mathcal{A}_{r}(z) & =\sum_{k \geq 0} a_{r+k, 0} \sum_{m \geq 0} s(m, k) \frac{z^{m}}{m !} \\
& =\sum_{k \geq 0} a_{r+k, 0} \frac{(\ln (1+z))^{k}}{k !} \\
& =\mathcal{B}_{r}(\ln (1+z)) .
\end{aligned}
$$

Example 3. A derangement on a set $\{1,2, \ldots, m\}$ is a permutation $\pi=i_{1} i_{2} \cdots i_{m}$ such that $i_{k} \neq k$ for $k=1,2, \ldots m$. The number of derangements on $\{1,2, \ldots, m\}$ is denoted by $D_{m}$ and given by $D_{m}=\left[\frac{m !}{e}\right]$, where $[x]$ the nearest integer function. 
Now, if the initial sequence $a_{0, m}=(-1)^{m} D_{m}$, then we get the following matrix

$$
8=\left(\begin{array}{ccccccc}
1 & 0 & 1 & -2 & 9 & -44 & \cdots \\
0 & 1 & 0 & 3 & -8 & 45 & \\
1 & 1 & 3 & 1 & 13 & -39 & \\
1 & 4 & 7 & 16 & 13 & 76 & \\
4 & 11 & 30 & 61 & 128 & 159 & \\
11 & 41 & 121 & 311 & 671 & 1381 & \\
\vdots & & & & & &
\end{array}\right)
$$

The generating function of the sequence $a_{0, m}$ is $A_{0}(z)=\frac{e^{z}}{1+z}$. It follows from (4.1) that $B_{0}(z)=\exp \left(e^{z}-z-1\right)$, and we notice that $a_{n, 0}$ is the number $v_{n}$ of partitions of $\{1,2, \ldots, n\}$ without singletons (see for instance [8]). By (3.3), one can then deduce that

Corollary 4. For $n, m \in \mathbb{N}_{0}$, we have

$$
\sum_{k=0}^{m} s(m, k) v_{n+k}=\sum_{k=0}^{n}(-1)^{m+k}\left\{\begin{array}{l}
n+m \\
k+m
\end{array}\right\}_{m} D_{m+k} .
$$

If $m=0$, we get the well-known identity (cf. [9], p.1569)

$$
v_{n}=\sum_{k=0}^{n}(-1)^{k}\left\{\begin{array}{l}
n \\
k
\end{array}\right\} D_{k} .
$$

Example 4. The exponential generating function of the Bernoulli polynomials $B_{n}(x)$ is

$$
\mathcal{B}_{0}(z):=\frac{z e^{x z}}{e^{z}-1}=\sum_{n \geq 0} B_{n}(x) \frac{z^{n}}{n !} .
$$

By Theorem 4, we have

$$
\mathcal{A}_{0}(z)=\frac{(1+z)^{x} \ln (1+z)}{z}
$$

It is not difficult to show that

$$
\left[z^{m}\right] \mathcal{A}_{0}(z)=\sum_{i=0}^{m}(-1)^{m-i} \frac{(x)_{i}}{m-i+1},
$$

where $\left[z^{n}\right] f(z)$ denote the operation of extracting the coefficient of $z^{n}$ in the formal power series $f(z)=\sum f_{n} z^{n}$. Now, let us consider $\delta$ defined by (1.4) with the final sequence $a_{n, 0}=B_{n}(x)$, by (3.3), we obtain the following corollary 
Corollary 5. For $n, m \in \mathbb{N}_{0}$, we have

$$
\sum_{k=0}^{m} s(m, k) B_{n+k}(x)=\sum_{k=0}^{n}\left\{\begin{array}{l}
n+m \\
k+m
\end{array}\right\} \sum_{m}^{m+k}(-1)^{m+k-i} \frac{(x)_{i}}{m+k-i+1} .
$$

Notice that the Todorov theorem [10, Eq. 49] is obtained by setting $n=0$ in (4.3).

Example 5. Catalan and Motzkin numbers naturally appear in a large number of combinatorial objects. It is well known that the Catalan number $C_{n}=\frac{1}{n+1}\left(\begin{array}{c}2 n \\ n\end{array}\right)$ and Motzkin number $M_{n}=\sum_{k=0}^{\lfloor n / 2\rfloor} \frac{1}{k+1}\left(\begin{array}{c}n \\ 2 k\end{array}\right)\left(\begin{array}{c}2 k \\ k\end{array}\right)$ are connected by [1]

$$
C_{n+1}=\sum_{k=0}^{n}\left(\begin{array}{l}
n \\
k
\end{array}\right) M_{k} \Longleftrightarrow M_{n}=\sum_{k=0}^{n}(-1)^{n-k}\left(\begin{array}{l}
n \\
k
\end{array}\right) C_{k+1} .
$$

Using the generalized Stirling transform, we can show that the Catalan numbers are related to the Motzkin numbers in terms of Stirling numbers by

$$
\sum_{k=0}^{n} s(n, k) M_{k}=\sum_{k=0}^{n+1} s(n+1, k) C_{k}
$$

and

$$
C_{n}=\delta_{n, 0}+\sum_{k=1}^{n} \sum_{i=0}^{k-1}\left\{\begin{array}{l}
n \\
k
\end{array}\right\} s(k-1, i) M_{i} \Longleftrightarrow M_{n}=\sum_{k=0}^{n} \sum_{i=0}^{k+1}\left\{\begin{array}{l}
n \\
k
\end{array}\right\} s(k+1, i) C_{i} .
$$

Setting the final sequence $a_{n, 0}=C_{n}$, we get the following matrix

$$
8=\left(\begin{array}{ccccccccc}
1 & 1 & 1 & 1 & 0 & 1 & -5 & 29 & \cdots \\
1 & 2 & 3 & 3 & 1 & 0 & -1 & 7 & \\
2 & 5 & 9 & 10 & 4 & -1 & 1 & -1 & \\
5 & 14 & 28 & 34 & 15 & -4 & 5 & -11 & \\
14 & 42 & 90 & 117 & 56 & -15 & 19 & -42 & \\
42 & 132 & 297 & 407 & 209 & -56 & 72 & -160 & \\
132 & 429 & 1001 & 1430 & 780 & -208 & 272 & -614 & \\
\vdots & & & & & & & &
\end{array}\right)
$$

Since

$$
\mathscr{B}_{0}(z)=\sum_{n \geq 0} C_{n} \frac{z^{n}}{n !}={ }_{1} F_{1}\left(\begin{array}{c}
1 / 2 \\
2
\end{array} ; 4 z\right)
$$


where ${ }_{1} F_{1}\left(\begin{array}{l}p \\ q\end{array} ; z\right)$ denotes the Kummer confluent hypergeometric function defined by

$$
\sum_{n \geq 0} \frac{\langle p\rangle_{n}}{\langle q\rangle_{n}} \frac{z^{n}}{n !}
$$

It follows from (4.2) that

$$
\mathcal{A}_{0}(z)=\sum_{n \geq 0} R_{n} \frac{z^{n}}{n !}={ }_{1} F_{1}\left(\begin{array}{c}
1 / 2 \\
2
\end{array} ; 4 \ln (1+z)\right)
$$

and

$$
\sum_{k=0}^{m} s(m, k) C_{n+k}=\sum_{k=0}^{n}\left\{\begin{array}{l}
n+m \\
k+m
\end{array}\right\}_{m} R_{m+k} .
$$

Now, if the initial sequence $a_{0, m}=R_{m+1}$, we get the following matrix

$$
\mathcal{T}=\left(\begin{array}{ccccccccc}
1 & 1 & 1 & 0 & 1 & -5 & 29 & -196 & \cdots \\
1 & 2 & 2 & 1 & -1 & 4 & -22 & 146 & \\
2 & 4 & 5 & 2 & 0 & -2 & 14 & -100 & \\
4 & 9 & 12 & 6 & -2 & 4 & -16 & 93 & \\
9 & 21 & 30 & 16 & -4 & 4 & -3 & -26 & \\
21 & 51 & 76 & 44 & -12 & 17 & -44 & 172 & \\
51 & 127 & 196 & 120 & -31 & 41 & -92 & 282 & \\
\vdots & & & & & & & &
\end{array}\right)
$$

From this matrix we observe that $a_{n, 0}=M_{n}$. We prove this observation using generating functions. We have

$$
\begin{aligned}
A_{0}(z) & =\sum_{n \geq 0} R_{n+1} \frac{z^{n}}{n !} \\
& =\frac{d}{d z}\left(\sum_{n \geq 0} R_{n} \frac{z^{n}}{n !}\right) \\
& =\frac{1}{1+z}{ }_{1} F_{1}\left(\begin{array}{c}
3 / 2 \\
3
\end{array} ; \ln (1+z)\right) .
\end{aligned}
$$

From (4.1), we get

$$
\begin{aligned}
B_{0}(z) & ={ }_{1} F_{1}\left(\begin{array}{c}
3 / 2 \\
3
\end{array} ; 4\right) e^{-z} \\
& =\frac{d}{d z}\left(\sum_{n \geq 0} C_{n} \frac{z^{n}}{n !}\right) \sum_{n \geq 0}(-1)^{n} \frac{z^{n}}{n !}
\end{aligned}
$$




$$
\begin{aligned}
& =\sum_{n \geq 0}\left(\sum_{k=0}^{n}(-1)^{n-k}\left(\begin{array}{l}
n \\
k
\end{array}\right) C_{k+1}\right) \frac{z^{n}}{n !} \\
& =\sum_{n \geq 0} M_{n} \frac{z^{n}}{n !} .
\end{aligned}
$$

It follows

$$
\sum_{k=0}^{m} s(m, k) M_{n+k}=\sum_{k=0}^{n}\left\{\begin{array}{l}
n+m \\
k+m
\end{array}\right\}_{m} R_{m+k+1} .
$$

Combining results (4.6) and (4.7) gives (4.4) and (4.5).

\section{HANKEL TRANSFORM}

The Hankel transform of a sequence $\alpha_{n}$ is the sequence of Hankel determinants $\operatorname{det}\left(\alpha_{i+j}\right)_{0 \leq i, j \leq n}$. A number of methods for computing the Hankel determinants have been widely investigated [4,5]. It is well known that the Hankel transform of sequences $\alpha_{n}$ and $\beta_{n}$ are equal under the binomial transform [6]

$$
\beta_{n}=\sum_{k=0}^{n}\left(\begin{array}{l}
n \\
k
\end{array}\right) \alpha_{k} .
$$

A natural question arises: "What about the Hankel transform of the sequences $a_{n}$ and $b_{n}$ under the Stirling transform?" In this section we show that there is a connection between the generalized Stirling transform and the Hankel determinants.

Theorem 5. For $n \in \mathbb{N}_{0}$, we consider a matrix $\left(a_{i, j}\right)_{0 \leq i, j \leq n}$ of order $n$ arising from (1.3) with initial sequence $a_{0, j}$ and the final sequence $b_{i}:=a_{i, 0}$, then

$$
\operatorname{det}\left(a_{i, j}\right)_{0 \leq i, j \leq n}=\operatorname{det}\left(b_{i+j}\right)_{0 \leq i, j \leq n},
$$

where $\operatorname{det}\left(b_{i+j}\right)_{0 \leq i, j \leq n}$ is the Hankel transform of the sequence $\left(b_{i}\right)$.

Proof. We can write

$$
\operatorname{det}\left(b_{i+j}\right)_{0 \leq i, j \leq n}=\left|\begin{array}{cccccc}
a_{0,0} & a_{1,0} & a_{2,0} & a_{3,0} & \cdots & a_{n, 0} \\
a_{1,0} & a_{2,0} & a_{3,0} & a_{4,0} & \cdots & a_{n+1,0} \\
\vdots & \vdots & \vdots & & \ddots & \vdots \\
a_{n, 0} & a_{n+1,0} & a_{n+2,0} & a_{n+3,0} & \cdots & a_{2 n, 0}
\end{array}\right|,
$$

after applying (1.4), the determinant is unchanged

$$
\operatorname{det}\left(b_{i+j}\right)_{0 \leq i, j \leq n}=
$$


$\left|\begin{array}{ccccc}a_{0,0} & a_{1,0} & a_{2,0}-a_{1,0} & a_{3,0}-a_{2,0}-2\left(a_{2,0}-a_{1,0}\right) & \ldots \\ a_{1,0} & a_{2,0} & a_{3,0}-a_{2,0} & a_{4,0}-a_{3,0}-2\left(a_{3,0}-a_{2,0}\right) & \ldots \\ \vdots & \vdots & \vdots & \vdots & \\ a_{n, 0} & a_{n+1,0} & a_{n+2,0}-a_{n+1,0} & a_{n+3,0}-a_{n+1,0}-2\left(a_{n+2,0}-a_{n+1,0}\right) & \ldots\end{array}\right|$.

Using (3.2), we get

$$
\operatorname{det}\left(b_{i+j}\right)_{0 \leq i, j \leq n}=\left|\begin{array}{cccccc}
a_{0,0} & a_{0,1} & a_{0,2} & a_{0,3} & \cdots & a_{0, n} \\
a_{1,0} & a_{1,1} & a_{1,2} & a_{1,3} & \cdots & a_{1, n} \\
\vdots & \vdots & \vdots & & \ddots & \\
a_{n, 0} & a_{n, 1} & a_{n, 2} & a_{n, 3} & & a_{n, n}
\end{array}\right|,
$$

from which the relation follows.

The answer to the previous question is given in the following

Corollary 6. For $n \in \mathbb{N}_{0}$, we have

$$
\operatorname{det}\left(b_{i+j}\right)_{0 \leq i, j \leq n}=\operatorname{det}\left(\sum_{k=0}^{i}\left\{\begin{array}{l}
i+j \\
k+j
\end{array}\right\}_{j} a_{k+j}\right)_{0 \leq i, j \leq n} .
$$

\section{ACKNOWLEDGEMENT}

The author would like to thank an anonymous reviewer for careful reading of this manuscript and their constructive remarks.

\section{REFERENCES}

[1] F. R. Bernhart, "Catalan, Motzkin, and Riordan numbers.” Discrete Math., vol. 204, no. 1-3, pp. 73-112, 1999.

[2] M. Bernstein and N. Sloane, "Some canonical sequences of integers." Linear Algebra Appl., vol. 226-228, pp. 57-72, 1995.

[3] A. Z. Broder, "The $r$-Stirling numbers." Discrete Math., vol. 49, pp. 241-259, 1984.

[4] C. Krattenthaler, "Advanced determinant calculus: a complement." Linear Algebra Appl., vol. 411, pp. 68-166, 2005.

[5] C. Krattenthaler, "Advanced determinant calculus.” Sémin. Lothar. Comb., vol. 42, pp. b42q, 67, 1999.

[6] J. W. Layman, "The Hankel transform and some of its properties.” J. Integer Seq., vol. 4, no. 1, pp. art. 01.1.5, 11, 2001.

[7] M. Rahmani, "The Akiyama-Tanigawa matrix and related combinatorial identities." Linear Algebra Appl., vol. 438, no. 1, pp. 219-230, 2013.

[8] Y. Sun and X. Wu, "The largest singletons of set partitions." Eur. J. Comb., vol. 32, no. 3, pp. 369-382, 2011.

[9] Y. Sun, X. Wu, and J. Zhuang, "Congruences on the Bell polynomials and the derangement polynomials," J. Number Theory, vol. 133, no. 5, pp. 1564-1571, 2013.

[10] P. G. Todorov, "On the theory of the Bernoulli polynomials and numbers," J. Math. Anal. Appl., vol. 104, no. 2, pp. 309-350, 1984. 
Author's address

Mourad Rahmani

USTHB, Faculty of Mathematics, P.O. Box 32, El Alia,16111, Algiers, Algeria

E-mail address: mrahmani@usthb.dz 